\begin{tabular}{|cc|}
\hline HARDWOOD & BIRCH \\
BURNING & PYROLYSIS \\
METHOXYPHENOL & PARTICLES \\
PHENOXY & RADICALS \\
HEALTH & PROTECTION \\
\hline
\end{tabular}

Open access submitted manuscript version of The Science of the Total Environment 277 (2001) 69-75

Link to publisher: doi:10.1016/50048-9697(00)00863-9

\title{
Phenolic antioxidants in wood smoke
}

Jennica Kjällstrand and Göran Petersson

The phenolic antioxidants were also studied in alder smoke for meat curing

chimney emissions from residential wood burning smoke from burning of forest plant materials 


\title{
Phenolic antioxidants in wood smoke
}

\author{
Jennica Kjällstrand and Göran Petersson* \\ Department of Chemical Environmental Science, Chalmers University of Technologv, 41296 Göteborg. Sweden
}

\begin{abstract}
Ten prominent dimethoxyphenols were determined in birchwood smoke from choked and open laboratory burning, and in chimney smoke from a tiled stove. The structures of the methoxyphenols are similar to those of the well-known tocopherol and ubiquinol antioxidants. The dimethoxyphenols characterising hardwood smoke are stronger antioxidants than the corresponding 2-methoxyphenols present mainly in softwood smoke. The antioxidant activity is highest for the 2,6-dimethoxyphenols with 4-alkenyl and 4-alkyl groups, which constitute $60-70 \%$ of the total amount. Phenolic antioxidants are scavengers of oxygen radicals and should be considered when health hazards of small-scale incomplete biomass burning are estimated.
\end{abstract}

Keywords: Combustion, air pollutants, gas chromatography, methoxyphenols, phenoxy radicals, lipid peroxidation

*Corresponding author. Tel: +46-31-7722998; fax: +46-31-7722999

E-mail address: goranp@kmv.chalmers.se (G. Petersson) 


\section{Introduction}

The impact on health of wood smoke is currently an issue of great concern with respect to small-scale burning of wood as a renewable fuel. Particle-related health hazards, attributed mainly to polycyclic organic compounds, were assessed in a large American study in Boise, Idaho (Cupitt et al., 1994). The carcinogens benzene and 1,3-butadiene are prominent among the emitted volatile hydrocarbons (Barrefors and Petersson, 1995). An important but often overlooked fact is that most of the organic compounds emitted from incomplete burning are related in structure to the lignin and carbohydrates of the biomass (Alén et al., 1996). These compounds are therefore very different from the better known hazardous compounds in combustion emissions from stationary and mobile sources. Analytical data have previously been given for lignin-specific methoxyphenols in smoke from forest biomass burning (Kjällstrand et al., 1998; Kjällstrand et al., 2000). Methoxyphenols were early identified as prominent thermal degradation products of wood (Faix et al., 1990) and as semi-volatile components of wood smoke particles (Hawthorne et al., 1989).

Phenols possess basic antioxidant properties (Simic, 1992). The antioxidant activities of the phenolic tocopherols and ubiquinols have been extensively studied (Barclay et al., 1990; Niki, 1997) because of their crucial importance in living organisms. Interest in flavonoids and a variety of other plant phenols is rapidly growing because of their antioxidant effects (Ogata et al., 1997) and potential prevention of health hazards (Leanderson et al., 1997; Newmark, 1996; Tsuda et al., 1994). Antioxidant properties have also recently been demonstrated for several lignin-related methoxyphenols (Barclay et al., 1997; Ogata et al., 1997). Our communication relates the antioxidant properties of specific assessed methoxyphenols to the impact of wood smoke on human health. The discussion is based on the formation of methoxyphenols from birchwood burning. 


\section{Methods}

Laboratory burning of birchwood was performed in a ceramic pot (1.5 1) placed in a fume cupboard. Smoke from choked burning was collected by placing a clay pot up-side down over the fire after flaming burning of about half of the amount of wood. Smoke samples were withdrawn through the bottom hole of the clay pot, using a gas-tight syringe. Smoke samples from open burning were taken $50 \mathrm{~cm}$ above a similar pot fire with a conventional air pump during the whole period of flaming burning. Chimney samples of smoke from burning in a tiled stove were taken in the same way in the chimney pipe outlet. In Table 1, comparative data are given for the three different types of burning experiments.

All samples were taken on injector liner glass cartridges $(0.8 \mathrm{~mm}$ i.d.) with the porous polymer Tenax TA (60-80 mesh) as adsorbent. Thermal desorption at $220^{\circ} \mathrm{C}$ was accomplished in the injector of the gas chromatograph (Varian 3800). The separations were made on a fused silica open tubular column $(30 \mathrm{~m} \times 0.25 \mathrm{~mm}$ i.d. $)$ with a cyanopropylphenylsilicone ( $\operatorname{Rtx} 1701,0.25 \mu \mathrm{m}$ layer) as the stationary phase. The oven temperature was increased $5^{\circ} \mathrm{C} \min ^{-1}$ from $40^{\circ} \mathrm{C}$ to $240^{\circ} \mathrm{C}$. Helium was used for desorption and as carrier gas. The column was linked to a Varian Saturn 2000 ion trap mass spectrometer. Electron impact mass spectra $(70 \mathrm{eV}, \underline{m / z} 35-400)$ were recorded. Concentrations were calculated assuming the same response for all the structurally similar dimethoxyphenols (Kjällstrand et al., 2000). 


\section{Results and discussion}

\subsection{Methoxyphenols in smoke}

This study was focussed on the 2,6-dimethoxyphenols, which have especially strong antioxidant effects. They are major components of hardwood smoke and are formed by degradation of hardwood lignin. The chromatogram in Fig. 1 illustrates the separation of the ten most prominent 2,6-dimethoxyphenols in smoke from choked burning of birchwood. All these species contain the 4-hydroxy-3,5-dimethoxyphenyl (syringyl, Sy) group, which characterises the phenylpropane building blocks of hardwood lignin. The alkyl, alkenyl, and carbonyl-containing groups of the phenols are remainders of the original $\mathrm{C}_{3}$ chain in lignin.

The results in Table 1 compare concentrations and proportions of the ten 2,6dimethoxyphenols for three widely differing burning conditions. The concentrations in chimney smoke from stove burning and in smoke from open laboratory burning are similar. The very high concentrations from choked laboratory burning are partly explained by the enclosure of smoke on choking. Several statistically significant differences in relative proportions of the ten phenols are likely to be due to the differing burning parameters in a complex way. The higher temperature for open laboratory and tiled stove burning results in higher proportions of the chemically least reactive $\mathrm{SyH}, \mathrm{SyCH}_{3}$ and $\mathrm{SyCH}_{2} \mathrm{CH}_{3}$ species. A low proportion of glowing for the open laboratory burning of small sticks may be related to the observed lower proportions of the carbonyl-containing phenols.

Birchwood smoke also contains smaller amounts of 2-methoxyphenols from corresponding 4-hydroxy-3-methoxyphenyl (guaiacyl) units in the lignin. The guaiacyl compounds elute earlier than the corresponding syringyl compounds on gas chromatography (Kjällstrand et al., 1998), but those with high retention appear in Fig. 1 as small peaks. Smoke 


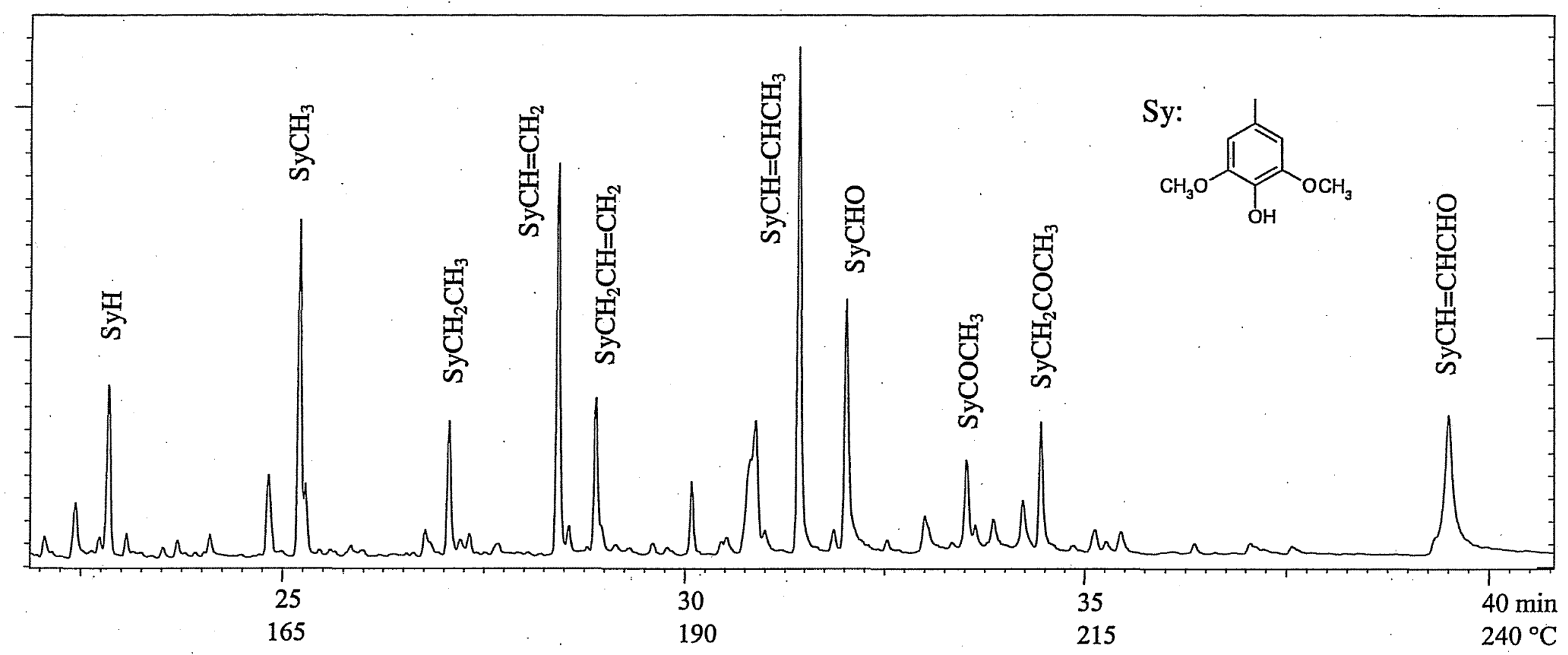

Fig. 1. Total ion chromatogram of a smoke sample from choked birchwood burning, illustrating the separation and the proportions of the ten most prominent dimethoxyphenol antioxidants. 


\section{Table 1}

Antioxidants in smoke from birchwood burning. Total concentrations and percentage proportions of the ten most prominent 4-hydroxy-3,5-dimethoxyphenyl (syringyl, Sy) compounds, arranged in order of estimated decreasing antioxidant strength.

\begin{tabular}{lccc}
\hline Burning & Choked in laboratory & Open in laboratory & In tiled stove \\
Firewood & Match-size sticks & Match-size sticks & Sticks and lumps \\
Weight $(\mathrm{g})$ & 2 & 5 & 500
\end{tabular}

Sampling

Volume (ml)

Time (min)

Number of samples

Total conc. $\left(\mathrm{mg} \mathrm{m}^{-3}\right)$
From covered pot

2

0.5

6

$146 \pm 72$
Above pot fire, $50 \mathrm{~cm}$

40

2

6

$9.1 \pm 7.7$

$4.0 \pm 1.6$

Proportions (\%, average \pm standard deviation)

\begin{tabular}{|c|c|c|c|}
\hline $\mathrm{SyCH}=\mathrm{CHCH}_{3}(\underline{E})$ & $22.4 \pm 2.3$ & $14.1 \pm 1.6$ & $14.0 \pm 2.3$ \\
\hline $\mathrm{SyCH}=\mathrm{CH}_{2}$ & $13.0 \pm 1.4$ & $15.2 \pm 1.2$ & $5.5 \pm 1.3$ \\
\hline $\mathrm{SyCH}_{2} \mathrm{CH}=\mathrm{CH}_{2}$ & $5.9 \pm 0.7$ & $5.6 \pm 0.6$ & $5.7 \pm 0.8$ \\
\hline $\mathrm{SyCH}_{2} \mathrm{CH}_{3}$ & $5.4 \pm 0.9$ & $8.9 \pm 0.8$ & $10.0 \pm 1.4$ \\
\hline $\mathrm{SyCH}_{3}$ & $11.0 \pm 1.7$ & $21.9 \pm 1.9$ & $19.1 \pm 2.6$ \\
\hline $\mathrm{SyCH}_{2} \mathrm{COCH}_{3}$ & $6.1 \pm 0.4$ & $3.5 \pm 0.7$ & $9.8 \pm 1.2$ \\
\hline $\mathrm{SyCH}=\mathrm{CHCHO}(E)$ & $12.4 \pm 0.9$ & $5.1 \pm 1.3$ & $5.6 \pm 0.6$ \\
\hline SyH & $6.7 \pm 1.4$ & $15.5 \pm 2.1$ & $12.0 \pm 3.5$ \\
\hline $\mathrm{SyCOCH}_{3}$ & $4.8 \pm 0.7$ & $2.8 \pm 0.3$ & $6.0 \pm 0.9$ \\
\hline SyCHO & $12.3 \pm 1.9$ & $7.3 \pm 2.0$ & $12.3 \pm 3.5$ \\
\hline
\end{tabular}


from other hardwood species than birch may contain larger proportions of guaiacyl compounds (Hawthorne et al., 1989). Softwood smoke contains 2-methoxyphenols in high concentrations (Kjällstrand et al., 2000) but not 2,6-dimethoxyphenols because of a different lignin structure.

The applied different burning conditions all correspond to incomplete combustion with large emissions of methoxyphenols. Increased combustion temperature not only efficiently decreases the emissions of organic compounds but also dramatically decreases the proportion of methoxyphenols (Alén et al., 1996). Methoxyphenols are the major organic products of lignin pyrolysis up to about $800^{\circ} \mathrm{C}$, but are almost negligible at $1000^{\circ} \mathrm{C}$. Smoke from average residential burning still contains appreciable proportions of methoxyphenols (Hawthorne et al, 1989). New devices for residential wood burning at higher temperatures are likely to eliminate emissions of methoxyphenols efficiently.

\subsection{Antioxidant properties}

As illustrated in Fig. 2, the structural features of the methoxyphenols in wood smoke are similar to those of well-known physiological phenolic antioxidants such as ubiquinols and tocopherols. The antioxidant properties are due to oxidation of the phenols to comparatively non-reactive phenoxy radicals (Simic, 1992). Methyl (alkyl) and methoxyl (alkoxyl) groups in ortho and para position stabilise the phenoxy radicals further and strengthen the antioxidant properties. The stabilising effect of a methoxyl group is stronger than that of a methyl group according to reported redox potentials for phenols with these substituents (Lind et al., 1990; Simic, 1992). The hardwood phenols with two radical-stabilising methoxyl groups are expected to be more efficient antioxidants than the softwood phenols with only one methoxyl 


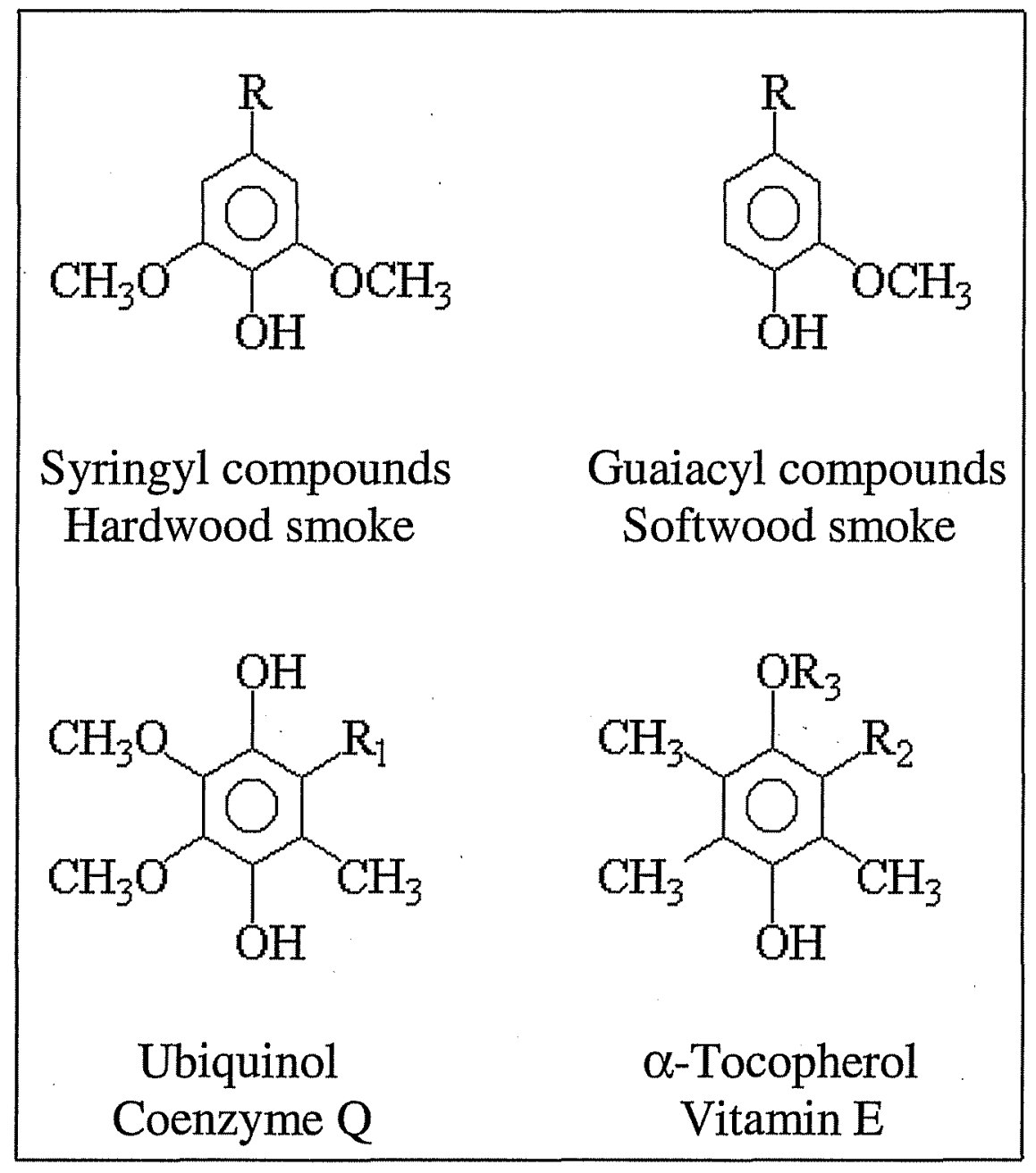

Fig. 2. Structural relationships between wood smoke methoxyphenols, ubiquinol and $\alpha-$ tocopherol. 
group. Using two different in vitro antioxidant activity assays, such a difference has been clearly demonstrated between the two methoxyphenols with a 2-propenyl (allyl) group in the para position (Barclay et al., 1997; Ogata et al., 1997).

In the living cell, the antioxidants complement the enzymatic defence against oxygen radicals. Tocopherols and other phenolic antioxidants react as scavengers of peroxide radicals, thereby preventing extensive peroxidation of lipids and other essential biomolecules (Barclay et al., 1990; Niki, 1997). The single phenolic hydroxyl group of the methoxyphenols makes them similar to $\alpha$-tocopherol as antioxidants. Ubiquinols differ by two hydroxyl groups in para position and are therefore easily autoxidised, resulting in the formation of oxygen radicals and a decreased antioxidant effect (Niki, 1997). The methoxyphenols in wood smoke lack the long hydrocarbon side chains of $\alpha$-tocopherol and human ubiquinol. By analogy with ubiquinols of differing chain length (Niki, 1997), this is likely to make the methoxyphenols more easily mobile within and between cell organelles.

Specific differences in antioxidant activity between the ten 2,6-dimethoxyphenols in Table 1 can be predicted. The conjugated double bonds of $\mathrm{SyCH}=\mathrm{CH}_{2}$ and $\mathrm{SyCH}=\mathrm{CHCH}$ stabilise the phenoxy radical, resulting in especially high antioxidant activity for these prominent smoke components. A clear difference for $\mathrm{GuCH}=\mathrm{CHCH}_{3}$, relative to $\mathrm{GuCH}_{2} \mathrm{CH}=\mathrm{CH}_{2}$ with a non-conjugated double bond, has been demonstrated in different test systems (Barclay et al., 1997; Priyadarsini et al., 1998). The effect of the conjugated double bond is approximately as large as that of the additional methoxyl group in $\mathrm{SyCH}_{2} \mathrm{CH}=\mathrm{CH}_{2}$ (Barclay et al., 1997). The phenols with a $\mathrm{CH}_{2}$ group adjacent to the aromatic ring $\left(\mathrm{SyCH}_{3}\right.$, $\mathrm{SyCH}_{2} \mathrm{CH}_{3}, \mathrm{SyCH}_{2} \mathrm{CH}=\mathrm{CH}_{2}, \mathrm{SyCH}_{2} \mathrm{COCH}_{3}$ ) are expected to have similar activities, which are higher than that of SyH (Barclay et al., 1997; Ogata et al., 1997, Lind et al., 1990). The electron-withdrawing $\alpha$-carbonyl groups of $\mathrm{SyCHO}$ and $\mathrm{SyCOCH}_{3}$ destabilise the phenoxy radical, resulting in lowered antioxidant activity (Lind et al., 1990; Barclay et al., 1997). The 
carbonyl group of $\mathrm{SyCH}=\mathrm{CHCHO}$ offsets the effect of the conjugated double bond (Barclay et al., 1997). It should be observed that the $\alpha$-carbonyl group also lowers the $\mathrm{pK}_{\mathrm{a}}$ of the phenols to approximately 7.4 for the aldehydes SyCHO and GuCHO (Ragnar et al., 1999). Possibly, these phenols exist as phenolate ions in blood. Phenolate ions are normally stronger antioxidants than the corresponding phenols (Simic, 1992).

The 2,6-dimethoxyphenols have two bulky groups adjacent to the phenolic hydroxyl group, which is generally recognised as kinetically favourable for chain-breaking reactions in cellular lipid peroxidation (Barclay et al., 1990). The effect is linked to decreased hydrogen bonding for phenols with bulky ortho groups and is therefore observed in aqueous systems. Investigated methoxyphenols have strong chain-braking antioxidant activity both in organic solutions (Barclay et al., 1997) and in aqueous micelle test systems (Ogata et al., 1997). It is concluded that 2,6-dimethoxyphenols are likely to counteract propagation of cellular lipid peroxidation more efficiently than antioxidants with similar redox potentials but without bulky ortho groups. The additional methoxyl group should also be kinetically favourable compared with corresponding 2-methoxyphenols.

Another important aspect of antioxidants is their ability to scavenge superoxide radicals, which initiate radical chain reactions. Electron spin trapping studies have demonstrated methoxyphenols to be scavengers of superoxide radicals (Ogata et al., 1997). The only 2,6dimethoxyphenol included, $\mathrm{SyCH}_{2} \mathrm{CH}=\mathrm{CH}_{2}$, was the most efficient scavenger. Superoxide trapping ability essentially parallelled lipid peroxidation inhibition for a range of different phenols. The ionic superoxide radical occurs in the aqueous phase, requiring sufficient water solubility of antioxidants in order to be efficient scavengers. The solubility of the methoxyphenols is intermediate between those of the lipid-soluble $\alpha$-tocopherol and the water-soluble ascorbic acid. Evidently this permits efficient trapping of both superoxide 
radicals and lipid peroxide radicals. Differences in solubility between specific methoxyphenols may cause differences in phase distribution and antioxidant activity.

\subsection{Health aspects}

Exposure to wood smoke phenols represents antioxidant intake by breathing, which is very different from the extensively studied dietary intake. The antioxidative properties of a variety of phenolic compounds in food may counteract basic health problems such as cell ageing, development of atherosclerosis, and initiation of cancer (Niki 1997; Ogata et al., 1997; Newmark, 1996). Recent interest has focussed on flavonoids and other dietary antioxidants with two or more phenolic hydroxyl groups. Many of these prevent genotoxic effects by scavenging hydrogen peroxide (Nakayama, 1994) but may also release counteracting superoxide radicals similarly as ubiquinols (Brusick, 1993; Niki, 1997). The effects are therefore different from those of monophenols, which trap radicals without release of reactive species. Vanillin, 4-hydroxy-3-methoxybensaldehyde, has been demonstrated to have a chemopreventive effect in a carcinogenicity test (Tsuda et al., 1994). Vanillin is probably the weakest methoxyphenol antioxidant in wood smoke, and stronger effects may be anticipated for all the dimethoxyphenols reported here. This is of particular interest with respect to lung cancer, because the respiratory tissues are directly exposed to the wood smoke components on inhalation.

The assessed dimethoxyphenols appear condensed on particles in ambient wood smoke (Hawthorne et al., 1989). The comprehensive Boise campaign estimated the lifetime lung cancer risk due to extractable organic matter from particles (Cupitt et al., 1994). For the same amount of extracted material, the risk was found to be about ten times larger for particles from 
mobile sources than for particles from residential wood burning. The antioxidant effects of the methoxyphenols in the lungs are likely to explain much of this striking difference. Evidently, the different impact on health of wood smoke particles compared to particles from other sources should be considered when emissions from traditional small-scale wood burning are regulated.

In addition to their antioxidative effect, phenols are also potential trapping agents for electrophilic genotoxic compounds (Newmark, 1996). Inhibition effects of phenols, including methoxyphenols, have been observed for the carcinogenic benso(a)pyrene (Wattenberg et al., 1980). Related polycyclic aromatic compounds are regarded as responsible for the lung cancer effect of urban particulate air pollution (Cupitt et al., 1994). Chemical interactions in the lungs between methoxyphenols and polycyclic aromatic compounds may explain part of the lower carcinogenicity of wood smoke particles as compared with particles from vehicle emissions.

The methoxyphenols are predominant thermal degradation products from lignin at temperatures below $800^{\circ} \mathrm{C}$. At higher temperatures, the emissions of methoxyphenols decrease rapidly, and the proportion of polycyclic aromatic hydrocarbons increases rapidly (Alén et al., 1996). The health effects of the methoxyphenol-rich smoke from open traditional wood burning are therefore expected to be very different from those of the emissions of modern high-temperature stove burning. It is also concluded that hardwood is preferable to softwood for open burning, with respect to the dimethoxyphenol content of the smoke.

\section{References}

Alén R, Kuoppala E, Oesch P. Formation of the main degradation compound groups from wood and its components during pyrolysis. J Anal Appl Pyrolysis 1996;36:137-148.

Barclay LRC, Baskin KA, Dakin KA, Locke SJ, Vinqvist, MR. The antioxidant activities in 
free radical peroxidation of phospholipid membranes. Can J Chem 1990;68:2258-2269.

Barclay LRC, Xi, F, Norris JQ. Antioxidant properties of phenolic lignin model compounds. J Wood Chem Techn 1997;17(1-2):73-90.

Barrefors G, Petersson G. Volatile hydrocarbons from domestic wood burning. Chemosphere $1995 ; 30: 1551-1556$.

Brusick D. Genotoxicity of phenolic antioxidants. Toxicol Ind Health 1993;9:223-230.

Cupitt LT, Glen WG, Lewtas J. Exposure and risk from ambient particle-bound pollution in an airshed dominated by residential wood combustion and mobile sources. Environ Health Persp 1994;102(4):75-84.

Faix O, Meier D, Fortmann I. Thermal degradation products of wood. Gas chromatographic separation and mass spectrometric characterization of monomeric lignin derived products. Holz Roh - Werkstoff 1990;48:281-285.

Hawthorne SB, Krieger MS, Miller DJ, Mathiason MB. Collection and quantitation of methoxylated phenol tracers for atmospheric pollution from residential wood stoves. Environ Sci Technol 1989;23:470-475.

Kjällstrand J, Ramnäs O, Petersson G. Gas chromatographic and mass spectrometric analysis of 36 lignin-related methoxyphenols from uncontrolled combustion of wood. J Chromatogr A 1998;824:205-210.

Kjällstrand J, Ramnäs O, Petersson G. Methoxyphenols from Scandinavian forest plant materials. Chemosphere 2000;41:735-741.

Leandersson P, Faresjö ÅO, Tagesson C. Green tea polyphenols inhibit oxidant-induced DNA strand breakage in cultured lung cells. Free Radic Biol Med 1997;23(2):235-242.

Lind J, Shen X, Eriksen TE, Merényi G. The one-electron reduction potential of 4-substituted phenoxyl radicals in water. J Am Chem Soc 1990; 112: 479-482. 
Nakayama T. Suppression of hydroperoxide-induced cytotoxicity by polyphenols. Cancer Res 1994;54:1991-1993.

Newmark HL. Plant phenolics as potential cancer prevention agents. In: Dietary phytochemicals in cancer prevention and treatment. Plenum Press, New York, 1996, pp. $25-34$

Niki E. Mechanisms and dynamics of antioxidant action of ubiquinol. Molec Aspects Med $1997 ; 18: 63-70$.

Ogata M, Hoshi M, Shimotohno K, Urano S, Endo T. Antioxidant activity of magnolol, honokiol, and related phenolic compounds. JAOCS 1997;74:557-562.

Priyadarsini KI, Guha SN, Rao MNA. Physico-chemical properties and antioxidant activities of methoxyphenols. Free Radic Biol Med 1998;24(6):933-941.

Ragnar M, Eriksson T, Reitberger T. Radical formation in ozone reactions with lignin and carbohydrate model compounds. Holzforchung 1999;53:292-298.

Simic MG. Antioxidant compounds: an overview. In: Davies KJA, editor. Oxidative damage and repair. Pergamon Press, New York, 1992, pp. 47-59.

Tsuda H, Uehara N, Iwahori Y, Asamoto M, ligo M, Nagao M, Matsumoto K, Ito M, Hirono I. Chemopreventive effects of $\beta$-carotene, $\alpha$-tocopherol and five naturally occurring antioxidants on initiation of hepatocarcinogenesis by 2-amino-3-methylimidazo[4,5flquinoline in the rat. Jpn J Cancer Res 1994;85:1214-1219.

Wattenberg, LW, Coccia JB, Lam LKT. Inhibitory effects of phenolic compounds on benzo(a)pyrene-induced neoplasia. Cancer Res 1980;40:2820-2823. 